aortic arch abnormalities, pulmonary thick expansion can also be used to establish cardiopulmonary bypass through the femoral artery. The APW was closed through aortic way in order to reveal the edge of the defect, the aortic valve and coronary artery. Accurate preoperative prediction of pulmonary artery pressure, conscious sedation, hyperventilation slightly, vasodilating agent and vasoactive drugs are of great important in preventing pulmonary hypertension crisis.

\section{GW23-e1801 SURGICAL TREATMENT OF FIVE PATIENTS WITH INTERRUPTED AORTIC ARCH AND AORTOPULMONARY WINDOW}

doi:10.1136/heartjnl-2012-302920p.3

Wang Dong, Liu Yinglong, Su Junwu, Cheng Pei, Zhang Jing, Fan Xiangming, Li Zhiqiang, Ding Wenhong, Han Ling, Han Ling. Department of Pediatric Cardiology, Capital Medical University affiliated Beijing Anzhen Hospital

Objectives To summarised review the surgical experience of single stage repair of interrupted aortic arch (IAA) associated with aortopulmonary window (APW) in five patients.

Methods Between December 1997 and December 2009, five patients (male 4, female 1) diagnosed IAA with APW were surgically repaired. The operative age was 1.7 months- 13 years (median age, 1.6 years) old and body weight was $4.7-26 \mathrm{~kg}$. Three patients were IAA type A and 1 type B as well as 1 type C. Two patients were APW type I and 3 type III. The associated anomalies included aortic origin of the right pulmonary artery, subvalvular aortic stenosis, mitral regurgitation and atrial septal defect. Extracorporeal circulation was intubed through patent ductus arteriosus in three cases and femoral artery associated with ascending aorta in two cases. Aortic arch was rebuilded by profound hypothermia circulation arrest and end-to-side anastomosis directly or broadly with pericardium. Ascending aorta was blocked highly and APW was patched after cardioplegia.

Results One case died of pulmonary hypertension crisis. There were one case of tracheotomy and one case recurred severe pulmonary hypertension at average 22 months follow-up.

Conclusions IAA associated APW is a rare congenital cardiac anomaly that accounts for high mortality in infant because of progressive pulmonary hypertension. Once definite diagnosis, primary single stage repair should be used as the first choice, and will achieve high successful rate and good long-term prognosis. Ultrasound cardiogram, cardiovascular CT and angiocardiography can reduce misdiagnosis. A median sternotomy incision was made, and the entire arch vessels were mobilised. Cardiopulmonary bypass was instituted between a single venous cannula in the right atrial appendage and a single arterial cannula in the distal portion of the ascending aorta. Such as complex 